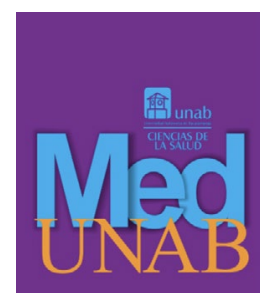

REVISTA DE LA FACULTAD

DE CIENCIAS DE LA SALUD

\title{
Xantoastrocitoma pleomórfico anaplásico y glioblastoma epitelioide: misma entidad o precursor de enfermedad
}

Anaplastic pleomorphic xanthoastrocytoma and epithelioid glioblastoma: same entity or precursor of disease

Xantoastrocitoma pleomórfico anaplásico e glioblastoma epitelioide: mesma entidade ou precursor de doença

María Alejandra Baquero-Serrano, MD., Esp. ${ }^{1}$ (D), Federico Guillermo LubinusBadillo, MD., Esp. ${ }^{1}$ (D), Silvia Nathalia Vera-Campos, $M D .^{2}$ (D)

1. Médico, Especialista en Radiología e Imágenes Diagnósticas. Servicio de Radiología, Clínica FOSCAL. Floridablanca, Santander, Colombia.

2. Médico, Residente de Radiología e Imágenes Diagnósticas. Universidad Autónoma de Bucaramanga. Floridablanca, Santander, Colombia.

Correspondencia. María Alejandra Baquero Serrano. Facultad de Ciencias de la Salud, Universidad Autónoma de Bucaramanga. Radiólogos Especializados de Bucaramanga S.A. Floridablanca, Santander, Colombia. Email. mbaquero@unab.edu.co

\section{INFORMACIÓN DEL ARTÍCULO:}

Artículo recibido: 11 de mayo de 2020

Artículo aceptado: 19 de noviembre de 2021

DOI: https://doi.org/10.29375/01237047.3905

Cómo citar. Baquero-Serrano MA, Lubinus-Badillo FG, Vera-Campos SN. Xantoastrocitoma pleomórfico anaplásico y glioblastoma epitelioide: misma entidad o precursor de enfermedad. MedUNAB [Internet]. 2021;24(3):359364. doi: https://doi.org/10.29375/01237047.3905

\section{RESUMEN}

Introducción. El xantoastrocitoma pleomórfico es una lesión glial de bajo grado de malignidad (grado II), puede presentar transformación maligna progresando a xantoastrocitoma pleomórfico anaplásico o glioblastoma multiforme, clasificados en grado III y IV, respectivamente, de acuerdo con la OMS. El glioblastoma epitelioide 
es un subtipo morfológico poco común del glioblastoma, de comportamiento agresivo, asociado a recurrencia temprana y compromiso leptomeníngeo. Presentación del caso. Se describe un reporte de caso de paciente femenina de 13 años con hallazgos de xantoastrocitoma pleomórfico anaplásico asociado a glioblastoma epitelioide, neoplasia poco frecuente que suele presentarse en la población pediátrica y en los adultos jóvenes. Discusión. El diagnóstico de glioblastoma epitelioide constituye un desafío, solo se han reportado unas pocas series pequeñas en la población adulta y pediátrica. Conclusión. Los hallazgos imagenológicos en las dos entidades son similares y comparten características histopatológicas e incluso algunos hallazgos moleculares superpuestos, lo cual dificulta su diferenciación, por lo que continúa siendo de gran controversia si se presentan conjuntamente o si el xantoastrocitoma pleomórfico anaplásico es un precursor del glioblastoma epitelioide.

Palabras clave:

Glioblastoma; Astrocitoma; Glioma; Neoplasias Encefálicas; Diagnóstico Diferencial.

\begin{abstract}
Introduction. Pleomorphic xanthoastrocytoma is a glial lesion with low grade of malignancy (grade II), it can present malignant transformation progressing to anaplastic pleomorphic xanthoastrocytoma or glioblastoma multiforme, classified as grade III and IV, respectively, according to the WHO. Epithelioid glioblastoma is a rare morphological subtype of glioblastoma, with aggressive behavior, associated with early recurrence and leptomeningeal compromise. Case Presentation. Case report of a 13-year-old female patient with findings of anaplastic pleomorphic xanthoastrocytoma associated with epithelioid glioblastoma, a rare neoplasm that usually occurs in the pediatric population and in young adults. Discussion. The diagnosis of epithelioid glioblastoma is challenging, only a few small series have been reported in the adult and pediatric population. Conclusion. The imaging findings in the two entities are similar and share histopathological characteristics and even some overlapping molecular findings, which makes their differentiation difficult. For this reason, there is still a great controversy whether these entities are present continuously or whether the anaplastic pleomorphic xanthoastrocytoma is a precursor of epithelioid glioblastoma.
\end{abstract}

Keywords:

Glioblastoma; Astrocytoma; Glioma; Brain Neoplasms; Diagnosis, Differential

\title{
RESUMO
}

Introdução. O xantoastrocitoma pleomórfico é uma lesão glial de baixo grau de malignidade (grau II), pode apresentar transformação maligna progredindo para xantoastrocitoma pleomórfico anaplásico ou glioblastoma multiforme, classificados como grau III e IV, respectivamente, de acordo com a OMS. O glioblastoma epitelióide é um subtipo morfológico raro de glioblastoma, com comportamento agressivo, associado a recorrência precoce e envolvimento leptomeníngeo. Apresentação do caso. É descrito um relatório de caso de uma paciente feminina de 13 anos com achados de xantoastrocitoma pleomórfico anaplásico associado ao glioblastoma epitelióide, uma neoplasia rara que geralmente ocorre na população pediátrica e em adultos jovens. Discussão. O diagnóstico do glioblastoma epitélioide é desafiador, apenas algumas pequenas séries foram reportadas na população adulta e pediátrica. Conclusão. As descobertas imagiológicas nas duas entidades são semelhantes e compartilham características histopatológicas e, até mesmo, algumas descobertas moleculares sobrepostas, o que dificulta sua diferenciação, portanto permanece controverso se ocorrem juntas ou se o xantoastrocitoma pleomórfico anaplásico é um precursor do glioblastoma epitélioide.

Palavras-chave:

Glioblastoma; Astrocitoma; Glioma; Neoplasias Encefálicas; Diagnóstico Diferencial.

\section{Introducción}

El xantoastrocitoma pleomórfico (XAP) fue descrito inicialmente por Kepes y colaboradores en 1979 (1). Constituye una lesión glial de bajo grado de malignidad, grado II según la Organización Mundial de la Salud (OMS), generalmente localizada en el córtex superficial, principalmente supratentorial, y en particular con afectación del lóbulo temporal, es más frecuente en niños y adultos jóvenes $(2,3)$. Presenta un 
pronóstico favorable; sin embargo, se ha propuesto su transformación maligna, la cual puede ocurrir entre un 10 y $20 \%$ de los casos dentro de un periodo de siete meses a 15 años, progresando a XAP anaplásico o glioblastoma multiforme, clasificados en grado III y IV, respectivamente, según la OMS (4).

Los Glioblastomas (GBM) pueden comprometer cualquier estructura neuroanatómica, en adultos es más común en los hemisferios cerebrales, mientras que en los niños lo es en la fosa posterior (5). Su crecimiento infiltrativo es extremadamente rápido. Se ha definido el GBM epitelioide (eGBM) como una variante provisional de GBM, con un comportamiento agresivo y generalmente similar a otros GBM (6).

Se presenta un caso clínico de una paciente en edad pediátrica que mostró hallazgos clínicos e imagenológicos de xantoastrocitoma pleomórfico vs glioblastoma multiforme, con el fin de describir las principales características de estas dos entidades y su relación, además de las diferentes estrategias diagnósticas para un diagnóstico oportuno.

\section{Presentación del caso}

Paciente femenina de 13 años con cuadro clínico de 12 días de evolución dado por cefalea holocraneal intensa, tinnitus y parestesias en extremidades, motivo por el cual consulta. En el examen físico se evidencian parestesias en extremidades y alteración del estado de conciencia. Es valorada por el servicio de neurocirugía por persistencia de sintomatología y se indica realización de resonancia magnética cerebral.

Se realiza estudio imagenológico con hallazgos de lesión expansiva heterogénea de tipo mixto con componente quístico y sólido de contornos regulares con focos múltiples de hipointensidad de señal en T1 e hiperintensidad en T2 (Figura 1), así mismo, se halla una zona de sangrado con hiperintensidad de señal en T1 y T2 hacia su aspecto anterior y caudal (Figura 1). No se observó captación del medio de contraste (Figura 1). Se identificó edema perilesional y efecto de masa con borramiento de los surcos pericerebrales (Figura 2), colapso del cuerno posterior del ventrículo lateral izquierdo y desviación de la línea media de izquierda a derecha. Del mismo modo, se visualizó restricción de la difusión (Figura 2). Dados los hallazgos en la resonancia cerebral se traslada a manejo quirúrgico y toma de biopsia para realizar diagnóstico de probable lesión de tipo neoplásico, procedimiento que se realizó sin ninguna complicación.
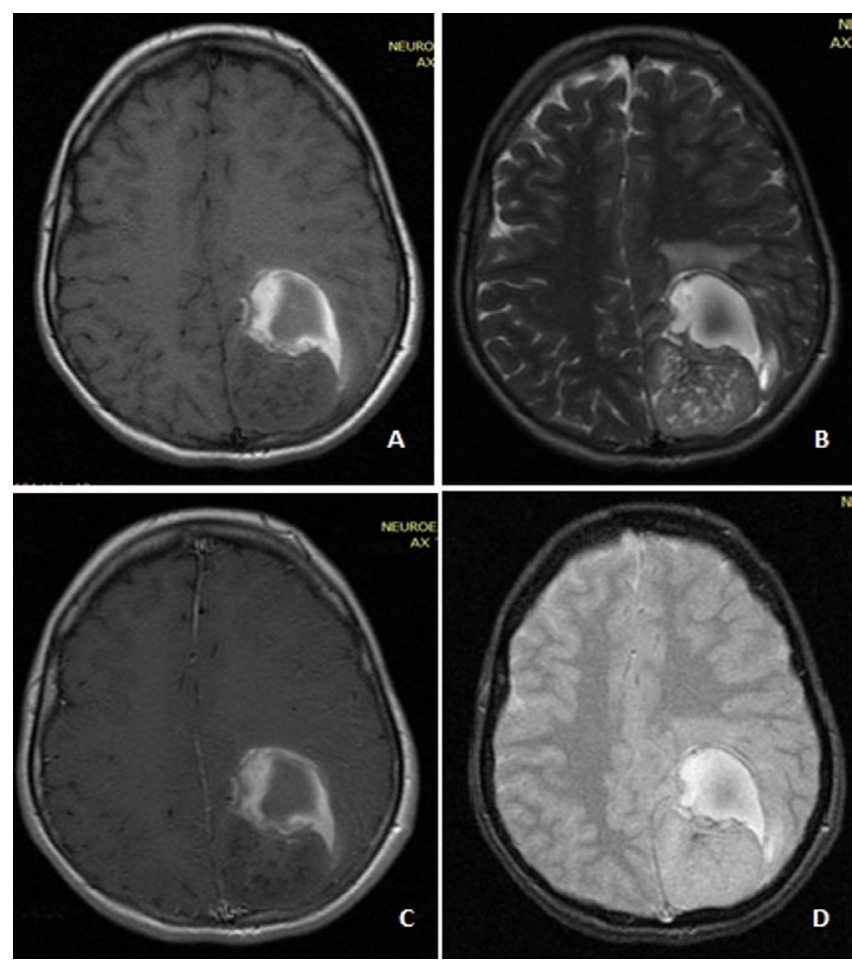

Figura 1. A) RMN Cerebral T1 Axial. Lesión heterogénea mixta de contornos irregulares hipointensa en T1. B) T2 Axial. Lesión heterogénea mixta de contornos irregulares hiperintensa en T2. C) T1 con contraste Axial. Lesión heterogénea mixta de contornos irregulares sin evidencia de captación de medio de contraste. D) Eco de gradiente Axial. Zona de sangrado hacia su aspecto anterior y caudal.

Fuente: elaborado por los los autores.
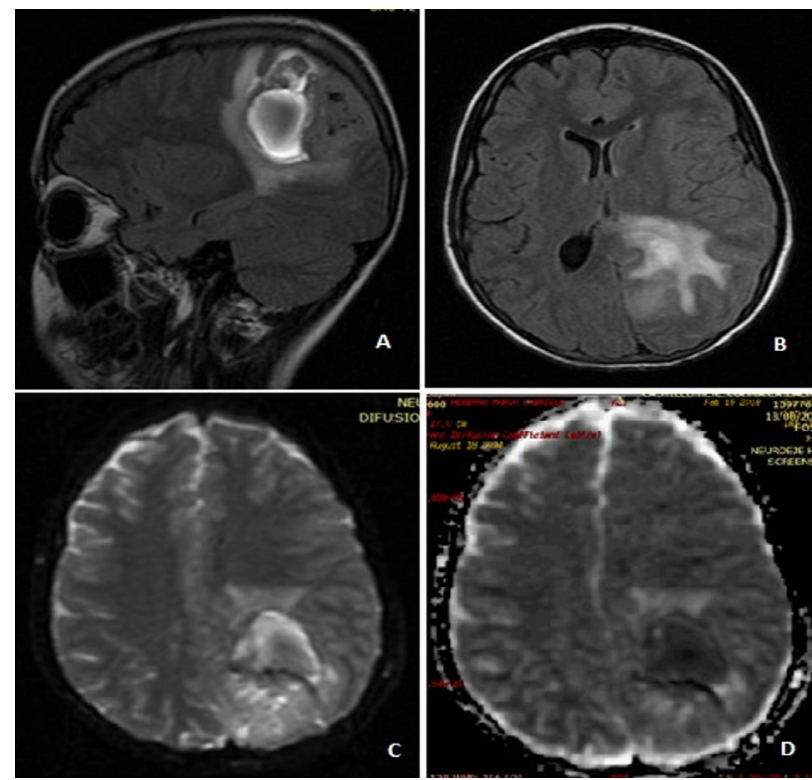

Figura 2. A) RMN Cerebral FLAIR Sagital. Edema perilesional de tipo vasogénico y efecto de masa con borramiento de los surcos pericerebrales. B) FLAIR Axial. Colapso del cuerno posterior del ventrículo lateral izquierdo y desviación de la línea media de izquierda a derecha. C y D) DWI - MAPA de ADC. Restricción de la difusión.

Fuente: elaborado por los los autores. 
El resultado histopatológico de lesión tumoral extraída muestra hallazgos de tumor glial de alto grado con áreas compatibles con xantoastrocitoma pleomórfico anaplásico (OMS grado III) y áreas compatibles con glioblastoma epitelioide (OMS grado IV).

\section{Discusión}

El xantoastrocitoma pleomórfico es un astrocitoma de bajo grado, con un pronóstico favorable después de la resección quirúrgica total. Ocurre principalmente en las primeras tres décadas de la vida, con un pico de incidencia en la segunda década (2). La localización suele ser predominantemente a nivel supratentorial, sobre todo en el lóbulo temporal y parietal, dada la ubicación hemisférica superficial de los XAP, la presentación clínica más común son las crisis epilépticas y el déficit neurológico focal, evento que ha sido reportado en el $70 \%$ de los pacientes (7).

Las características histológicas clásicas del XAP son la presencia de células pleomórficas gigantes, redondeadas por una red de reticulina, caracterizadas por la inclusión citoplasmática de vacuolas lipídicas. Los XAP pueden sufrir transformación maligna asociada principalmente con aumento de la actividad mitótica y necrosis (2). Radiológicamente, los hallazgos son inespecíficos, se evidencian lesiones hipointensas en $\mathrm{T} 1$ e hiperintensas en T2 con un componente interno quístico y sólido, acompañado de efecto de masa severo, edema vasogénico y afectación de las leptomeninges (8).

Presenta una tasa de recurrencia del $30 \%$ en 5 años y $40 \%$ en 10 años después de la resección total; sin embargo, se han descrito casos de progresión maligna de XAP hacia lesiones anaplásicas y astrocitomas malignos, incluyendo el glioblastoma multiforme. Actualmente, se conoce que entre el $15 \%$ y $20 \%$ de los XAP presentan rasgos anaplásicos (9), y existen 2 tipos: el XAP anaplásico primario (de novo), y el secundario, originado a partir de la transformación anaplásica que puede ocurrir en casos de recidivas tumorales de XAP de bajo grado. La causa y los mecanismos implicados en dicha transformación siguen sin esclarecerse, incluso es difícil de predecir tomando en cuenta las características morfológicas del tumor primario (10).

En 1983 Weldon-Linne et al (11) publicaron el primer caso de XAP anaplásico (XAP-A) en el contexto de una recidiva tumoral. El XAP-A, clasificado por la OMS como grado III, presenta un comportamiento más severo en intervalos de recurrencia y capacidad de metástasis; por lo tanto, requiere de una terapia más agresiva, este tipo de tumor puede hacer metástasis linfática $\mathrm{o}$ hematógena y puede presentarse como hemorragia intracerebral. Histológicamente se diferencia del XAP por la inclusión de cuerpos eosinofílicos granulares, cambios desmoplásicos, infiltración linfoide y su mayor grado de diferenciación neuronal (7).

Las características inmunohistoquímicas distintivas de los XAP-A son el índice mitótico elevado, la disminución de la positividad para marcadores de diferenciación neuronal y el aumento en el Ki67, que puede llegar hasta el 50\%. Adicionalmente, más de la mitad de los pacientes presentan la mutación V600E del BRAF y el $85 \%$ carece de la metilación del promotor MGMT (7).

El tratamiento de elección de los XAP es la resección quirúrgica, que al ser completa permite una supervivencia del $85 \%$ y $70 \%$ a los 5 y 10 años, respectivamente. En los $\mathrm{XAP}$ de bajo grado, la radioterapia y quimioterapia no presentan ningún beneficio; no obstante, en la variante anaplásica el uso de estas intervenciones modifica la supervivencia.

Por otro lado, el glioblastoma grado IV es uno de los tumores más malignos en la población adulta y pediátrica. La OMS ha reconocido diversos subtipos según variables morfológicas y moleculares $(12,13)$. La diferenciación epitelial se encuentra dentro de los patrones morfológicos más raros y solo es el $1.6 \%$ de todos los glioblastomas (13). Dentro de esta categoría se puede subdividir entre diferenciación pseudoepitelial (adenoide y epitelioide) y epitelial propiamente dicha (13). El Glioblastoma epitelioide es una de las variables más raras que fue adoptado en la clasificación de la OMS de 2016 (14).

El glioblastoma epitelioide tiene un curso agresivo y puede presentar recurrencia temprana, hemorragia intratumoral y diseminación leptomeníngea. Adicionalmente se caracteriza por la presencia de células tumorales epitelioides con abundante citoplasma eosinofílico, núcleo excéntrico y citología rabdoide, el $10 \%$ de los casos presentan vacuolas lipídicas en el citoplasma (13). Esto se acompaña, frecuentemente, de disminución de la cohesión celular, alta tasa mitótica $\mathrm{y}$ focos de necrosis tumoral con proliferaciones microvasculares (12).

El diagnóstico de glioblastoma epitelioide constituye un desafío y solo se han reportado unas pocas series pequeñas en la población adulta y pediátrica. Los tumores del SNC con características epitelioides son críticos y se ha observado que XAP-A tienen 
características patológicas similares, e incluso algunos hallazgos moleculares superpuestos. Del mismo modo que en el glioblastoma epitelioide, los XAP-A con transformación anaplásica afectan con mayor frecuencia a los niños y adultos jóvenes en concordancia con el caso presentado en paciente en edad pediátrica $(12,14)$.

Los hallazgos imagenológicos en las dos entidades son similares, se visualizan como una masa heterogénea de gran tamaño de márgenes irregulares, con áreas de necrosis quística y realce nodular, que se rodea por edema vasogénico con efecto de masa y compresión de estructuras adyacentes, hallazgos característicos en el presente caso acompañado de diseminación leptomeníngea. Dentro de los diagnósticos diferenciales se encuentran el glioblastoma, las metástasis cerebrales, el histiocitoma fibroso maligno, el astrocitoma anaplásico pilocítico y el tumor rabdoide/teratoide (14).

El glioblastoma epitelioide tiene frecuentemente mutaciones BRAF V600E entre el $16.6 \%$ y $93 \%$ de los casos, es por esto que en ocasiones puede mostrar características histológicas y genéticas superpuestas con el XAP-A, dado a sus características epitelioides y falta de características degenerativas. Aunque la clasificación de la OMS en el 2016 incorporó pruebas moleculares, el diagnóstico se basa en hallazgos histopatológicos (14).

\section{Conclusión}

Aún existen dudas acerca de la relación que existe entre el xantroastrocitoma pleomórfico anaplásico y el glioblastoma epitelioide. Los estudios han demostrado que la resonancia magnética ha contribuido a proporcionar información útil acerca del tamaño del tumor, la presencia de edema perilesional, necrosis, hemorragia y degeneración quística permiten caracterizar la agresividad del tumor. Sin embargo, el diagnóstico preciso de estos tumores continúa siendo un desafío según los criterios de clasificación de la OMS definidos en el 2016. Por lo tanto, se requieren más estudios que brinden herramientas útiles en la profundización del conocimiento en las bases biológicas y moleculares de cada uno de estos tumores para el desarrollo de estrategias de tratamiento más eficaces.

\section{Conflictos de intereses}

Los autores declaran no tener conflictos de intereses.

\section{Consideraciones éticas}

Para la publicación de este artículo se contó con la autorización del paciente por medio del consentimiento informado, en donde autoriza la publicación de los datos con fines académicos e investigativos, según la resolución 8430 de 1993.

\section{Referencias}

1. Kepes JJ, Rubinstein LJ, Eng LF. Pleomorphic xanthoastrocytoma: A distinctive meningocerebral glioma of young subjects with relatively favorable prognosis. A study of 12 cases. Cancer [Internet]. 1979;44(5):1839-1852. doi: https://doi. org/10.1002/1097-0142(197911)44:5\%3C1839::AIDCNCR2820440543\%3E3.0.CO;2-0

2. Patibandla MR, Nayak M, PurohitAK, Thotakura AK, Uppin M, Challa S. Pleomorphic xanthoastrocytoma with anaplastic features: A rare case report and review of literature with reference to current management. Asian J Neurosurg [Internet]. 2016;11(3):319. doi: https://doi.org/10.4103/1793-5482.144161

3. Choudry U, Khan SA, Resident C, Qureshi A, Bari E. Primary anaplastic pleomorphic xanthoastrocytoma in adults. Case report and review of literature. Int J Surg Case Rep [Internet]. 2016;27:183-188. doi: https://doi.org/10.1016/j.ijscr.2016.08.022

4. Roberti F, Baggenstos M. Intraventricular Anaplastic Pleomorphic Xanthoastrocytoma: Very Rare Localization and Early Recurrence of a Rare Tumor. Cureus [Internet]. 2018;10(5):e2665. doi: https://doi. org/10.7759/cureus.2665

5. Castañeda CA, Casavilca S, Orrego E, GarciaCorrochano P, Deza P, Heinike H, et al. Glioblastoma: análisis molecular y sus implicancias clínicas. Rev. Perú Med Exp Salud Pública [Internet]. 2015;32(2):316-25. doi: https://doi.org/10.17843/ rpmesp.2015.322.1627

6. Furuta T, Miyoshi H, Komaki S, Arakawa F, Morioka $\mathrm{M}$, Ohshima K, et al. Clinicopathological and genetic association between epithelioid glioblastoma and pleomorphic xanthoastrocytoma. Neuropathology [Internet]. 2018;38(3):218-227. doi: https://doi. org/10.1111/neup.12459

7. Castro CD, Jiménez E, Useche N, Hakim F, Bermúdez $\mathrm{S}$, Rojas L. Xantoastrocitoma pleomórfico anaplásico recurrente y respuesta a la combinación de carmustine y bevacizumab, un reporte de caso. Acta Neurol Colomb [Internet]. 2018;34(4):239-244. doi: https:// doi.org/10.22379/24224022220

8. Binesh F, Akhavan A, Navabii H. Pleomorphic xanthoastrocytoma with malignant transformation 
and multiple recurrences in an Iranian girl. Case Reports [Internet]. 2012;2012:bcr1220115372. doi: https://doi.org/10.1136/bcr.12.2011.5372

9. Otluoglu GD, Ozek M. A rare clinical presentation: a pleomorphic xanthoastrocytoma presenting with intracerebral haemorrhage and metastasizing vigorously-case report and review of the literature. Childs Nerv Syst [Internet]. 2019;35:355-362. doi: https://doi.org/10.1007/s00381-018-3960-1

10. Rodríguez-Mena R, Joanes-Alepuz V, BarbellaAponte R, Pérez-Valles A. Xantoastrocitoma pleomórfico con extensión intraventricular $\mathrm{y}$ transformación anaplásica en paciente adulto: caso clínico. Neurocirugía [Internet]. 2012;23(5):203-210. doi: https://doi.org/10.1016/j.neucir.2011.08.003

11. Weldon-Linne CM, Victor TA, Groothuis DR, Vick NA. Pleomorphic xanthoastrocytoma. Ultrastructural and immunohistochemical study of a case with a rapidly fatal outcome following surgery. Cancer [Internet]. 1983;52(11):2055-63. doi: https://doi. org/10.1002/1097-0142(19831201)52:11<2055::aidcncr2820521115>3.0.co; 2 -w
12. Alexandrescu S, Korshunov A, Lai SH, Dabiri S, Patil $\mathrm{S}, \mathrm{Li}$ R, et al. Epithelioid Glioblastomas and Anaplastic Epithelioid Pleomorphic Xanthoastrocytomas-Same Entity or First Cousins? Brain Pathol [Internet]. 2016;26:215-23. doi: https://doi.org/10.1111/ bpa.12295

13. Gasco J, Franklin B, Fuller GN, Salinas P, Prabhu S. Multifocal epithelioid glioblastoma mimicking cerebral metastasis: case report. Neurocirugía [Internet]. 2009;20(6). doi: https://doi.org/10.4321/ $\underline{\text { S1130-14732009000600004 }}$

14. Watanabe N, Ishikawa E, Kohzuki H, Sakamoto $\mathrm{N}$, Zaboronok A, Matsuda M, et al. Malignant transformation of pleomorphic xanthoastrocytoma and differential diagnosis: case report. BMC Neurol [Internet]. 2020;20:21. doi: https://doi.org/10.1186/ $\underline{\text { s12883-020-1601-2 }}$ 